05,06

\title{
Структура и диэлектрические свойства наноструктурированной керамики $\mathrm{Bi}_{1-x} \mathrm{Sm}_{x} \mathrm{FeO}_{3}$
}

\author{
() С.А. Садыков ${ }^{1}$, Н.М-Р. Алиханов ${ }^{1,2, \uparrow, ~ С . Н . ~ К а л л а е в ~}{ }^{2}$, М.Х. Рабаданов ${ }^{1}$, Д.К. Палчаев ${ }^{1}$, \\ Ж.Х. Мурлиева ${ }^{1}$, Р.М. Эмиров ${ }^{1}$ \\ ${ }^{1}$ Дагестанский государственный университет, \\ Махачкала, Россия \\ ${ }^{2}$ Институт фризики им. Х.И. Амирханова, ДНЦ РАН, \\ Махачкала, Россия \\ 『 E-mail: alihanov.nariman@mail.ru
}

Поступила в Редакцию 24 июня 2019 г.

В окончательной редакции 24 июня 2019 г.

Принята к публикациии 15 июля 2019 г.

Исследованы структура и диэлектрические свойства поликристаллических мультиферроиков системы $\mathrm{Bi}_{1-x} \mathrm{Sm}_{x} \mathrm{FeO}_{3}(x=0-0.2)$. $X$-ray дифракцией установлено, что в составе с $x=0.1$ наноструктурированного образца сосуществуют две фазы: ромбоэдрическая $R 3 c$ и орторомбическая Pbam. На температурных зависимостях диэлектрической проницаемости $\varepsilon^{\prime}(T)$ обнаружены четыре аномалии, характерные для фазовых превращений, в области температур $\sim 180^{\circ} \mathrm{C}, \sim 250^{\circ} \mathrm{C}, \sim 300^{\circ} \mathrm{C}$ и $T_{N} \sim 350^{\circ} \mathrm{C}$. Показано, что с увеличением концентрации самария диэлектрическая проницаемость $\varepsilon^{\prime}$ возрастает, a $\operatorname{tg} \delta$ уменьшается.

Ключевые слова: $\mathrm{BiFeO}_{3}$, диэлектрические свойства, синтез, кристаллическая структура.

DOI: 10.21883/FTT.2019.11.48413.530

\section{1. Введение}

Мультиферроики продолжают оставаться в сфере интересов исследователей в связи с широкими перспективами создания на их основе различных технологических устройств, таких как элементы памяти, преобразователи солнечной энергии, элементы микроэлектроники и спинтроники $[1,2]$. Наибольший интерес привлекает феррит висмута, $\mathrm{BiFeO}_{3}$ (BFO), один из немногих мультиферроиков, в котором сегнетоэлектричество и антиферромагнетизм сосуществуют в одной фазе с экстремально высокими температурами упорядочений (температура Кюри ТС $\sim 830^{\circ} \mathrm{C}$ и температура Нееля $\left.T_{N} \sim 370^{\circ} \mathrm{C}[3]\right)$. Уникальные природные особенности ВFО позволяют создавать на его основе магнитоэлектрические устройства, работающие при комнатной температуре. Однако функциональные возможности BFO ограничиваются присутствием примесных фаз и различного рода дефектов структуры, порождающих диэлектрические потери и токи утечки.

Изучению влияния замещения редкоземельными элементами $(\mathrm{Re}) \mathrm{A}$-узла $\mathrm{BFO}$ на диэлектрические свойства, посвящено достаточно много работ. Однако нет ясного понимания того, какие физические механизмы ответственны за формирование диэлектрических параметров при замещении. Принято считать, что на электрические свойства BFO, также как и на магнитные, влияет угол связи $\mathrm{Fe}-\mathrm{O}-\mathrm{Fe}$, поскольку он контролирует как магнитный обмен, так и орбитальное перекрытие между $\mathrm{Fe}$ и $\mathrm{O}$ $[4,5]$. Замещение $A$-узла ионами с меньшим ионным радиусом приводит к изменению угла связи $\mathrm{Fe}-\mathrm{O}-\mathrm{Fe}$. Так как ионный радиус большинства редкоземельных трехвалентных ионов меньше, чем у $\mathrm{Bi}^{3+}$, то такое замещение приведет к искажению кристаллической структуры, а как следствие к значительному изменению физических свойств BFO. Замещение ионов $\mathrm{Bi}^{3+}$ ионами $\mathrm{Sm}^{3+}$ (ионный радиус $\mathrm{Sm}^{3+}-1.08 \AA, \mathrm{Bi}^{3+}-1.17 \AA$ при координационном числе 8) приводит к искажению октаэдра $\mathrm{FeO}_{6}$, изменению угла $\mathrm{Fe}-\mathrm{O}-\mathrm{Fe}$ и длин связей $\mathrm{Bi} / \mathrm{Sm}-\mathrm{O}$, что также приводит к увеличению поляризуемости с последующим увеличением диэлектрической проницаемости и снижением диэлектрических потерь. Подобный характер поведения диэлектрических свойств обнаруживается у образцов, допированных $\mathrm{Sm}$ [6], Yb [7], Y [8], Gd [9], тогда как у образцов, допированных La $[10,11]$ и $\operatorname{Pr}[12]$ наблюдается обратная тенденция, т.е. увеличение концентрации допантов приводит к уменьшению диэлектрической проницаемости. Более того, в [13] показано, что $\varepsilon^{\prime}$ образцов $\mathrm{Bi}_{1-x} \mathrm{Sm}_{x} \mathrm{FeO}_{3}$ растет с увеличением концентрации $\mathrm{Sm}$ до $x=0.05$, и, наоборот, убывает при концентрациях $x=0.05-0.15$. При этом диэлектрические потери уменьшились у всех образцов. Таким образом, явной корреляции между диэлектрическими параметрами BReFO и ионным радиусом допанта Re не обнаруживается. На это указывают также результаты диэлектрических измерений $\mathrm{Bi}_{0.96} \mathrm{Re}_{0.04} \mathrm{FeO}_{3}(\mathrm{Re}=\mathrm{La}, \mathrm{Yb}$, $\mathrm{Sm}, \mathrm{Dy})[14]$, выполненных при комнатной температуре.

Несмотря на интенсивные исследования последних лет, проблема влияния допирования на диэлектрические свойства в BFO не решена. Большинством авторов диэлектрические свойства BFO изучены в частотном диапазоне до $1 \mathrm{MHz}$ и связываются, как правило, с максвелл-вагнеровской релаксацией и конечной проводимостью образца. В данной работе мы сообщаем о влиянии замещения Sm на структуру и диэлектрические 
Структурные параметры получены из уточнения Ритвельда рентгенограмм керамики $\mathrm{Bi}_{1-x} \mathrm{Sm}_{x} \mathrm{FeO}_{3} \mathrm{c} x=0,0.5,0.10,0.15$ и 0.20

\begin{tabular}{|c|c|c|c|c|c|c|c|}
\hline \multirow{3}{*}{$\begin{array}{c}\mathrm{Sm} \\
\text { concentration } \\
(x)\end{array}$} & \multicolumn{5}{|c|}{ Lattice Parameters $(\AA)$} & \multicolumn{2}{|c|}{ Crystal structure (\%) } \\
\hline & \multicolumn{2}{|c|}{$\begin{array}{l}\text { Rhombohedral } \\
\text { phase }(R 3 c)\end{array}$} & \multicolumn{3}{|c|}{$\begin{array}{l}\text { Orthorhombic phase } \\
\text { (Pbam) }\end{array}$} & \multirow[t]{2}{*}{$R 3 c$} & \multirow[t]{2}{*}{ Pbam } \\
\hline & $a, \AA$ & $c, \AA$ & $a, \AA$ & $b, \AA$ & $c, \AA$ & & \\
\hline 0 & 5.578 & 13.864 & - & - & - & 100 & - \\
\hline 0.05 & 5.574 & 13.807 & - & - & - & 100 & - \\
\hline 0.1 & 5.565 & 13.724 & 5.582 & 11.214 & 7.817 & 47.4 & 52.6 \\
\hline 0.15 & - & - & 5.557 & 11.206 & 7.826 & - & 100 \\
\hline 0.2 & - & - & 5.546 & 11.209 & 7.815 & - & 100 \\
\hline
\end{tabular}

свойства нанокристаллической керамики ВFО в широкой области температур и частот.

\section{2. Объекты и методы исследования}

Объектами исследования являлись керамические образцы $\mathrm{Bi}_{1-x} \mathrm{Sm}_{x} \mathrm{FeO}_{3}$ (BSFO) $(x=0-0.2)$, спрессованные из нанопорошков, синтезированных методом сжигания нитрат органических прекурсоров. Исходные реактивы $\mathrm{Bi}\left(\mathrm{NO}_{3}\right)_{3}, \mathrm{Fe}\left(\mathrm{NO}_{3}\right)_{3}$ и $\mathrm{Sm}\left(\mathrm{NO}_{3}\right)_{3}$ (чистота $>98 \%$ ), взятые в стехиометрическом молярном отношении, растворялась в дистиллированной воде. К смеси добавлялся глицин в соответствующей пропорции. Раствор перемешивался с одновременным выпариванием, в результате вспышки синтезировался нанопорошок. Дифракционный анализ и оценка дисперсности нанопорошка проводились на дифрактометре PANalytical Empyrean series 2 с использованием излучения $\mathrm{Cu}-\mathrm{K} \alpha \quad(\lambda=1.54056 \AA)$. Уточнение структуры проводилось методом Ритвельда с использованием программного обеспечения FULLPROF. Спектры комбинационного рассеяния при комнатной температуре были получены с использованием спектрометра Ntegra Spectra в диапазоне волн $100-800 \mathrm{~cm}^{-1}$ с длиной волны возбуждения $532 \mathrm{~nm}$. Изображения SEM образцов получены на сканирующем электронном микроскопе LEO-1450. Частотные зависимости диэлектрических свойств в диапазоне $1 \mathrm{kHz}-10 \mathrm{MHz}$ проводились с использованием измерителя LCR-78110G фирмы „Good Will Instrument Со“ в интервале температур $25-400^{\circ} \mathrm{C}$. Образцы для исследования изготавливались в форме плоского конденсатора с толщиной $\sim 1 \mathrm{~mm}$ и диаметром $\sim 6 \mathrm{~mm}$ с контактами из серебросодержащей пасты.

\section{3. Результаты и обсуждения}

\section{1. Исследования структуры}

Фазовый анализ показал, что исходный нанопорошок BFO содержит порядка $1 \%$ примесных фаз $\mathrm{Bi}_{2} \mathrm{Fe}_{4} \mathrm{O}_{9}$ и $\mathrm{Bi}_{25} \mathrm{FeO}_{39}$. У легированных самарием составов BSFO в диапазоне концентраций $0.05 \leq x \leq 0.2$ наблюдается смещение дифракционных пиков в сторону более высоких значений, что указывает на искажения решетки (рис. 1) из-за меньшего ионного радиуса самария. Составы BSFO $(x=0 ; 0.05)$ кристаллизуются в ромбоэдрической структуре с пространственной группой симметрии $R 3 c$. При концентрации самария $10 \%$ кристаллическая структура BSFO описывается двухфазной моделью: наряду с ромбоэдрической фазой $R 3 c$ формируется антиполярная орторомбическая Pbam фаза в соотношении $\sim 1: 1$ (см. таблица). Для составов с концентрациями $x=0.15$ и 0.2 кристаллическая структура BSFO успешно описывается однофазной моделью орторомбической структуры, предполагающей антиполярный характер смещения ионов в $A$ - и $B$-позициях перовскита $[5,15]$. Следует отметить, что в ряде работ $[5,12,16,17]$ сообщается, что $10 \%$ замещение $\mathrm{Bi}^{3+}$ на $\mathrm{Sm}^{3+}$ не приводит структурно фазовым переходам, которые возможны при более высоких концентрациях. Однако, уточнение рентгенограммы этого состава методом Ритвельда указывает на присутствие новой фазы Pbam. Стоит отметить, что изменения параметров элементарной ячейки в орторомбической фазе более существенны по сравнению с ромбоэдрической фазой.

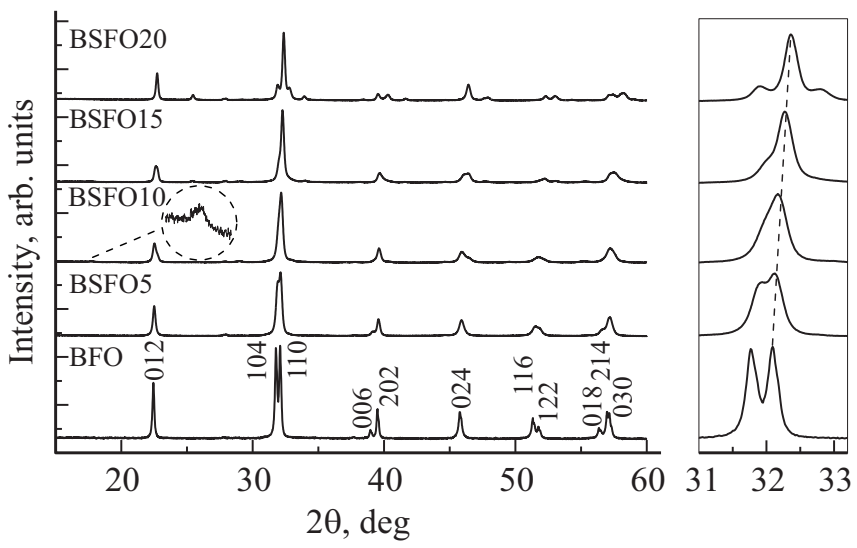

Рис. 1. Результаты дифракционного анализа образцов $\mathrm{Bi}_{1-x} \mathrm{Sm}_{x} \mathrm{FeO}_{3}(x=0 ; 0,05 ; 0,1 ; 0,15 ; 0,2)$. 


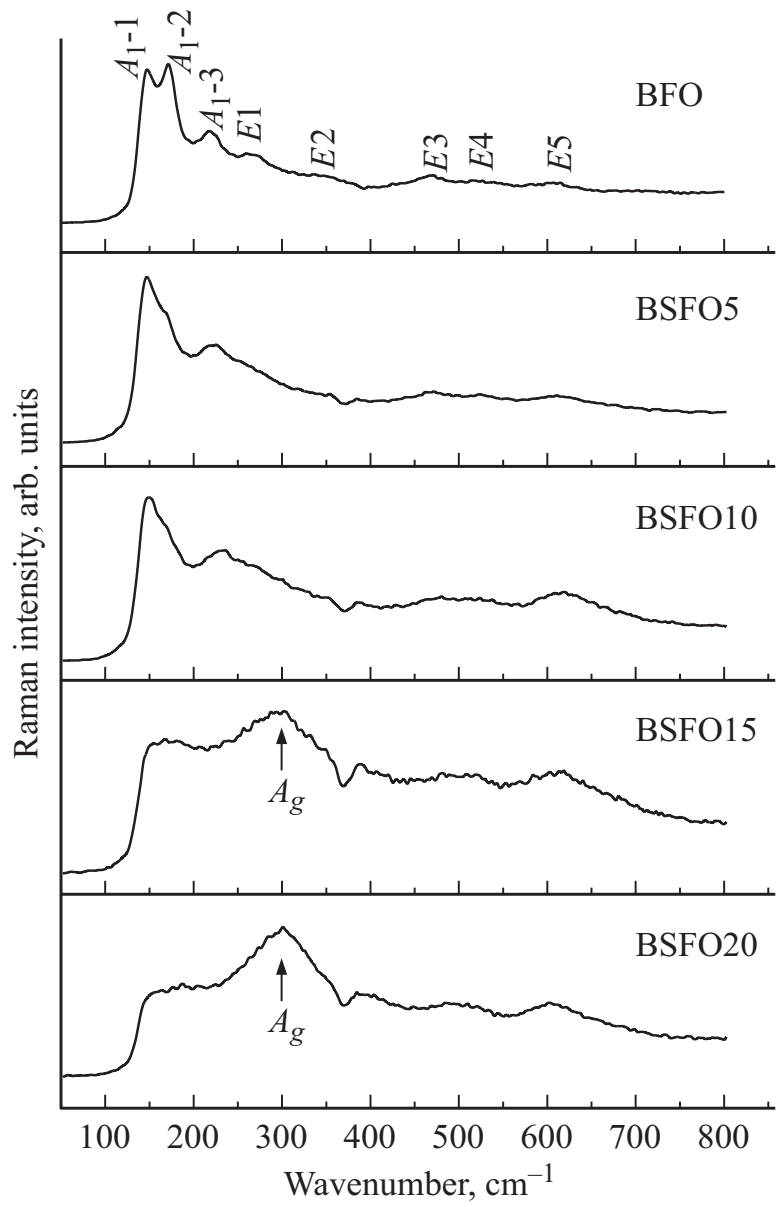

Рис. 2. Спектры комбинационного рассеяния образцов $\mathrm{Bi}_{1-x} \mathrm{Sm}_{x} \mathrm{FeO}_{3} \quad(x=0 ; 0,05 ; 0,1 ; 0,15 ; 0,2)$ при комнатной температуре.

Заметим также, что увеличение концентрации самария приводит к появлению новых пиков. Так, при $10 \%$ замещении появляется новый пик при $\sim 17.5^{\circ}$, а также наблюдается асимметрия пика $(012)$ при $\sim 22^{\circ}$, слияние пиков при $\sim 31.76^{\circ}(104)$ и $32.09^{\circ}(110)$. При $15 \%$ начинает проявляться пик (111) при $\sim 25^{\circ}$, характерный для орторомбической структуры $\mathrm{SmFeO}_{3}[15,18]$. Средний размер кристаллитов, вычисленный по формуле Шеррера, составляет $56 ; 40 ; 35 ; 32 ; 30 \mathrm{~nm}$ для $x=0 ; 0.05$; $0.1 ; 0.15$ и 0.2 , соответственно. С ростом доли самария наблюдается уменьшение размера кристаллитов.

Спектры комбинационного рассеяния для BSFO, снятые при комнатной температуре, представлены на рис. 2. Для ромбоэдрического $\mathrm{BFO}$ с $R 3 c$-пространственной группой характерно тринадцать $\left(4 A_{1}+9 E\right)$ активных мод [16]. Для состава $x=0$ наблюдалось восемь рамановских мод: $146\left(A_{1}-1\right), 171.67\left(A_{1}-2\right), 219.64\left(A_{1}-3\right)$, $258.16\left(E_{1}\right), 334\left(E_{2}\right), 466\left(E_{3}\right), 524.1\left(E_{4}\right), 608\left(E_{5}\right) \mathrm{cm}^{-1}$. При 10 процентном замещении $\mathrm{Sm}$ наиболее интенсивная мода $\left(A_{1}-1\right)$ сдвигается до $149 \mathrm{~cm}^{-1}$. Начиная с $10 \%$ замещения Sm спектры комбинационного рассеяния претерпевают существенные изменения, связанные с искажениями структуры. Данная мода $\left(149 \mathrm{~cm}^{-1}\right)$ полностью исчезает при концентрациях $\operatorname{Sm} x \geq 0.15$. Уширение рамановских мод с увеличением концентрации Sm соответствует уменьшению среднего размера кристаллитов, что согласуется с данными XRD. Для $x=0.15-0.2$ наблюдается широкий пик при $300 \mathrm{~cm}^{-1}$, связанный с $A_{g}$ модой, которая обусловлена вибрацией связей $\mathrm{Sm}-\mathrm{O}$ в орторомбической элементарной ячейке [19]. Мода $260 \mathrm{~cm}^{-1}$ ( $E$-мода), связанна с ковалентной связью $\mathrm{Fe}-\mathrm{O}$ и соответствующим углом связи $\mathrm{Fe}-\mathrm{O}-\mathrm{Fe}$. Моды свыше $600 \mathrm{~cm}^{-1}$ относятся к комбинационному рассеянию второго порядка, которое связано с электрон-фононным взаимодействием в BFO.

На рис. 3 показаны типичные SEM изображения образцов BFO и BSFO $(x=0.1$ и 0.2). Видно, что все образцы состоят из зерен с четко определенными границами,
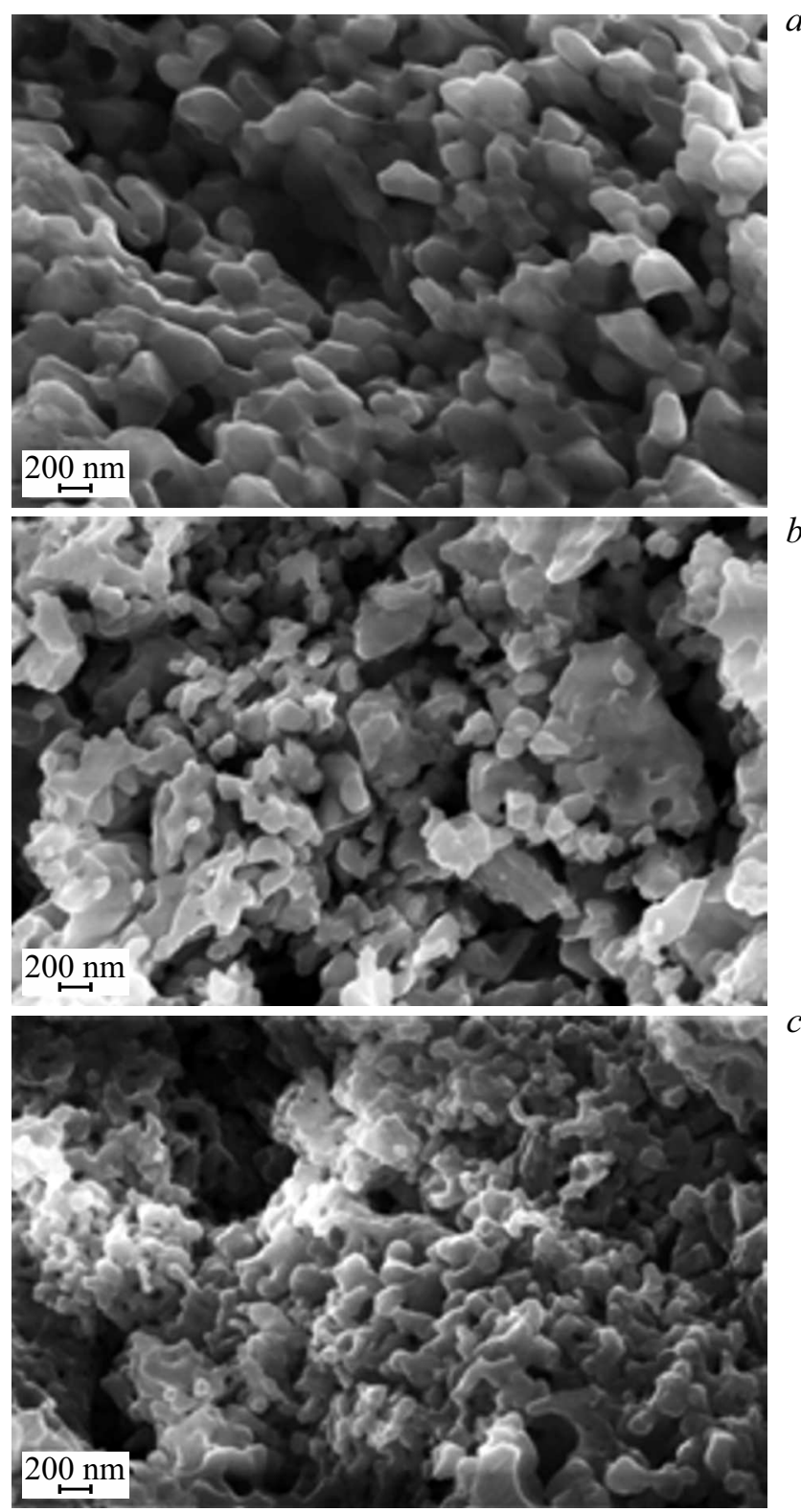

Рис. 3. СЭМ-изображения образцов $\mathrm{Bi}_{1-x} \mathrm{Sm}_{x} \mathrm{FeO}_{3} \quad$ c (a) $x=0,(b) x=0.1$ и (c) $x=0.2$. 
средние размеры которых составляют $\sim 200-300 \mathrm{~nm}$. При этом наблюдается тенденция к уменьшению размера зерен с увеличением концентрации самария.

\section{2. Диэлектрические свойства}

На рис. $4, a, b$ представлены результаты измерений частотных зависимостей реальной части $\varepsilon^{\prime}$ комплексной диэлектрической проницаемости и диэлектрических потерь $(\operatorname{tg} \delta)$ BFO и BSFO составов, выполненных при комнатной температуре. У всех образцов наблюдается значительная дисперсия диэлектрических свойств в исследованном диапазоне частот. Наибольшая дисперсия проявляется у BSFO20. Как и ожидалось, $\varepsilon^{\prime}$ и $\operatorname{tg} \delta$ монотонно уменьшаются с увеличением частоты и остаются почти неизменными на высоких частотах (свыше $10 \mathrm{kHz}$ ). В значения $\varepsilon^{\prime}$ могут давать вклады различные поляризационные механизмы, с различными временами релаксации. Образцы BFO и BSFO имеют мелкозернистую структуру с большим количеством межзеренных границ. В таких составах наибольший вклад в высокие значения $\varepsilon^{\prime}$ в области низких частот вносит межфазная поляризация объемного заряда. Вследствие блокировки на границах зерен и дефектах структуры, эти заряды, подобно диполям во внешнем электрическом поле, создают локальную поляризацию, известную как поляризация пространственного заряда [16]. Увеличение концентрации $\mathrm{Sm}$ приводит к большей неоднородности системы и появлению большего числа границ зерен (рис. 3), соответственно и накоплению большего пространственного заряда. Это приведет к увеличению явления релаксации пространственного заряда Максвелла-Вагнера в системе [20]. Следовательно, одной из вероятных причин высоких значений $\varepsilon^{\prime}$ у образцов с большей концентрации $\mathrm{Sm}$, особенно в области низких частот, является Максвелл-Вагнеровская релаксация пространственных зарядов, накапливающихся на границах зерен. С ростом частоты вклад межфазной поляризации, также как и процессов сегнетоэлектрических доменных переключений [21], постепенно снижается вследствие релаксационных процессов.

Как видно из рис. $4, a, b$, с увеличением процентного содержания самария $\varepsilon^{\prime}$ возрастает, а $\operatorname{tg} \delta$ уменьшается. При комнатной температуре на частоте $1 \mathrm{kHz}$ диэлектрическая проницаемость у BSFO20 ( $\left.\varepsilon^{\prime} \sim 150\right)$ почти в два раза выше, чем у $\mathrm{BFO}\left(\varepsilon^{\prime} \sim 80\right)$. Увеличение $\varepsilon^{\prime}$ BSFO15 и BSFO20 относительно BFO явно указывает на активную роль, которую играет $\mathrm{Sm}$ в изменение диэлектрических свойств. Отметим, что диэлектрические проницаемости составов BFO, BSFO5 и BSFO10 на частотах $>10 \mathrm{kHz}$ практически совпадают, тогда как у BSFO15 и BSFO20 существенно выше.

Температурные зависимости $\varepsilon^{\prime}$ и $\operatorname{tg} \delta$, измеренные на разных частотах для состава BSFO5, представлены на рис. 5, $a, b$. Оба параметра $\varepsilon^{\prime}(T)$ и $\operatorname{tg} \delta(T)$ проявляют характерную для релаксоров частотную зависимость. На зависимостях $\varepsilon^{\prime}(T)$ выделяются три явно выраженные аномалии, приходящиеся на различные температурные
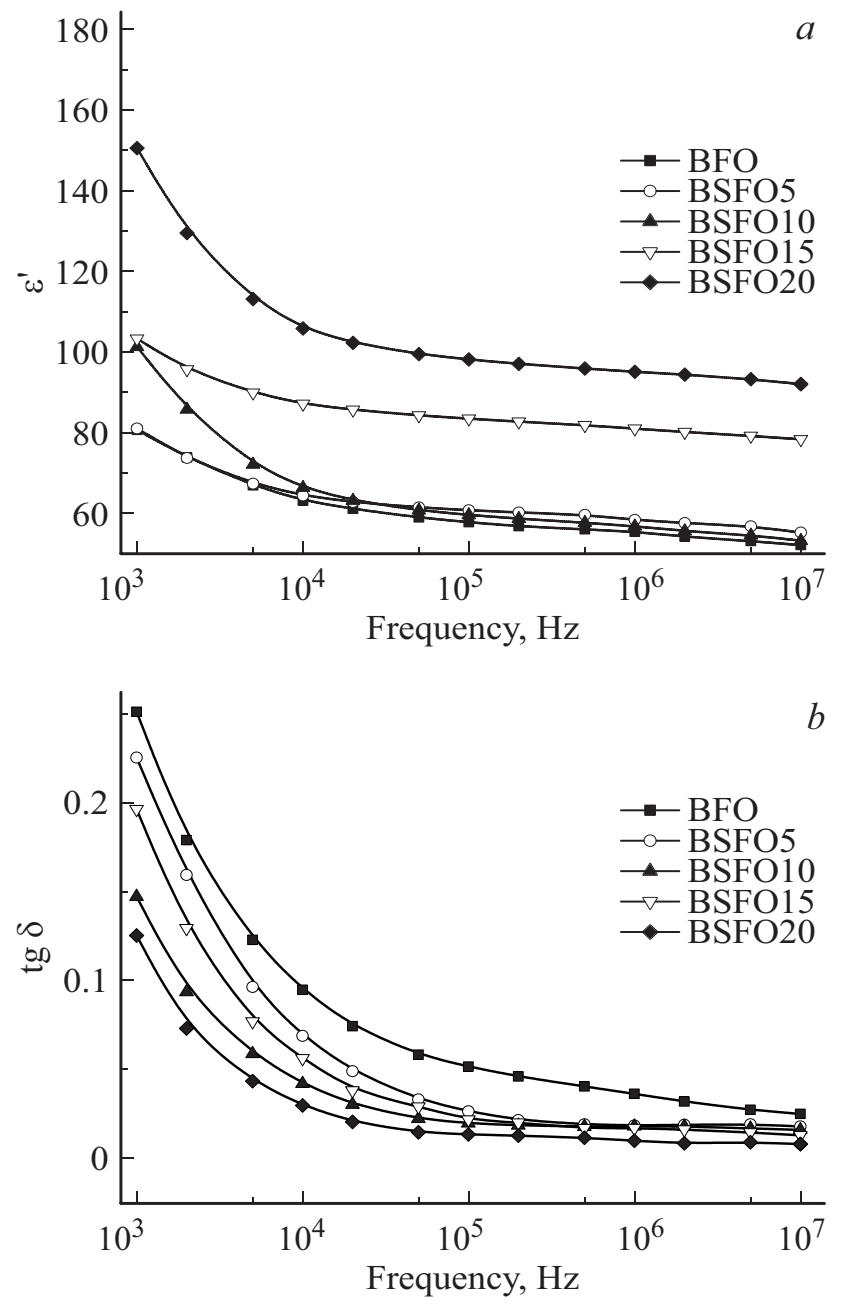

Рис. 4. Частотная зависимость $\varepsilon^{\prime}(a)$ и $\operatorname{tg} \delta(b)$ для образцов $\mathrm{Bi}_{1-x} \mathrm{Sm}_{x} \mathrm{FeO}_{3} \quad(x=0 ; 0,05 ; 0,1 ; 0,15 ; 0,2)$ при комнатной температуре.

участки (рис. 5,a). Первая, достаточно широкая, аномалия $\varepsilon^{\prime}(T)$ наблюдается в окрестности $\sim 240^{\circ} \mathrm{C}$, за которой после небольшого снижения следует резкое увеличение с пиком при $\sim 300^{\circ} \mathrm{C}$. Третий пик $\varepsilon^{\prime}(T)$ обнаруживается вблизи $\sim 350^{\circ} \mathrm{C}$, в окрестности температуры Нееля $T_{N}$.

Заметим, что зависимости $\varepsilon^{\prime}(T)$ для всех исследованных образцов в целом идентичны (рис. 6). Аномалии $\varepsilon^{\prime}(T)$ сильнее проявляются у составов с большей концентрацией самария (BSFO15, BSFO20), причем максимальные значения $\varepsilon^{\prime}\left(\sim 300^{\circ} \mathrm{C}\right)$ этих составов почти на порядок выше, чем у исходного. Диэлектрические потери также испытывают аномалии в окрестности указанных температур.

Еще одна аномалия диэлектрических свойств у исследованных нами образцов обнаруживается в интервале температур $T^{*} \sim 160-180^{\circ} \mathrm{C}$ (рис. 5, $\left.a, b\right)$. Об аномалии $\varepsilon^{\prime}(T)$ в мультиферроиках в области температур $\sim 140-240^{\circ} \mathrm{C}$ сообщается многими авторами $[8,10,14,19,21]$. Впервые диэлектрическая аномалия 


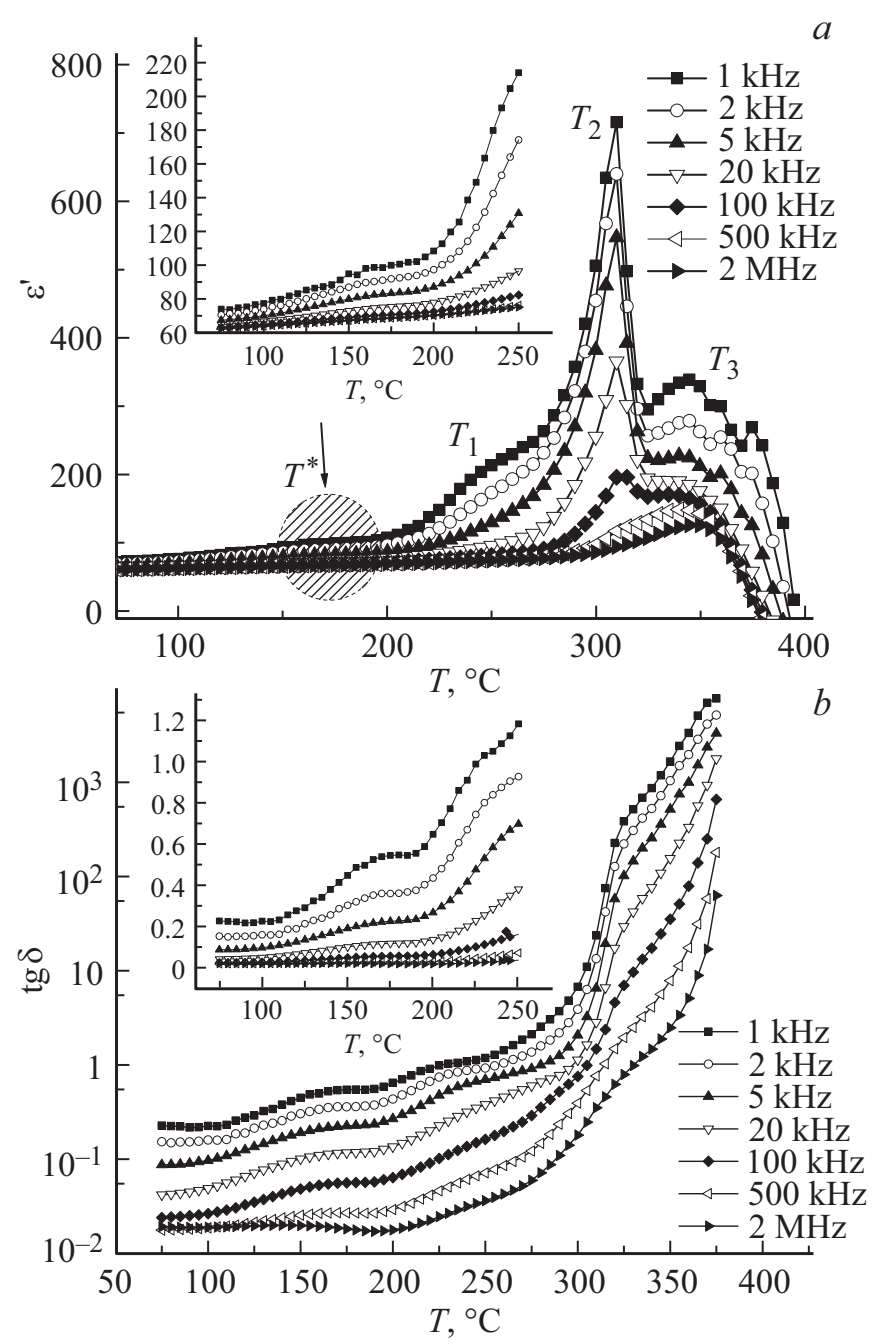

Рис. 5. Температурные зависимости $\varepsilon^{\prime}(a)$ и $\operatorname{tg} \delta(b)$ образца $\mathrm{Bi}_{0.95} \mathrm{Sm}_{0.05} \mathrm{FeO}_{3}$ на различных частотах.

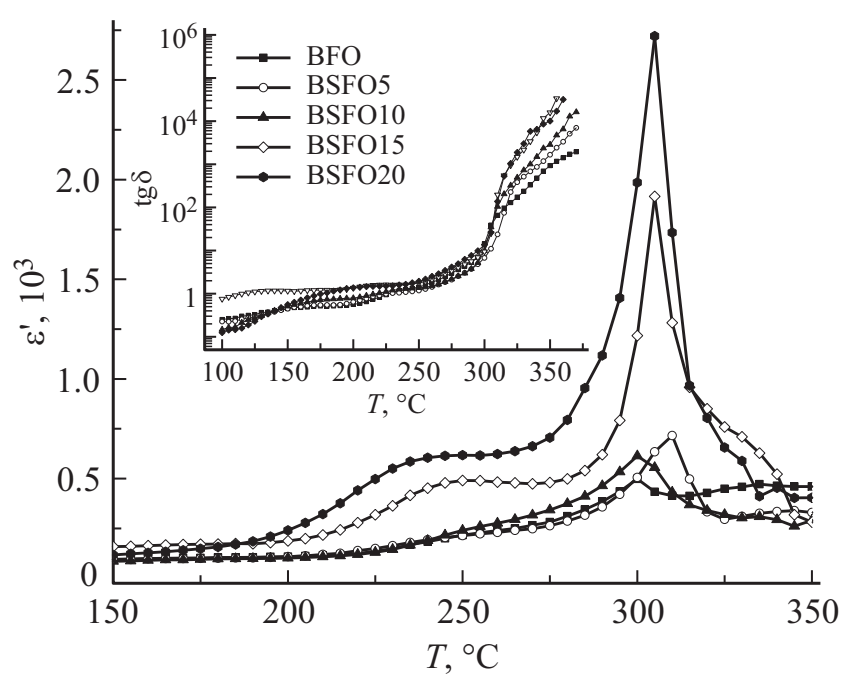

Рис. 6. Температурная зависимость $\varepsilon^{\prime}$ и $\operatorname{tg} \delta$ (вставка) образцов $\mathrm{Bi}_{1-x} \mathrm{Sm}_{x} \mathrm{FeO}_{3}(x=0 ; 0,05 ; 0,1 ; 0,15 ; 0,2)$ при частоте 1 кГц. для BFO, не связанная со структурными изменениями, была обнаружена Поломской [22] при температуре $458 \mathrm{~K}$. В $[6,10]$ считается, что аномалия $\varepsilon^{\prime}(T)$ может быть следствием (перезарядки ионов) переходного взаимодействия между вакансиями ионов кислорода и $\mathrm{Fe}^{3+} / \mathrm{Fe}^{2+}$ в окислительно-восстановительной реакции. Имеются и иные взгляды на переход Поломской: как результат „skin-effect“ в тонких пленках BFO [23], связь с углом $\mathrm{Fe}-\mathrm{O}-\mathrm{Fe}$ без изменения структурной симметрии [24], следствие аномального затухания спиновых волн в тонкой пленке BFO [25], спин-переориентации в наночастицах $\mathrm{SmFeO}_{3}$ [19].

Как видно из графиков температурных (рис. 6), как и частотных зависимостей (рис. 4) диэлектрической проницаемости для образцов BSFO15 и BSFO20 (орторомбическая Pbam фаза) наблюдаются относительно высокие значения $\varepsilon^{\prime}$ во всем частотном диапазоне.

\section{4. Заключение}

Исследованы структура и диэлектрические свойства керамики $\mathrm{Bi}_{1-x} \mathrm{Sm}_{x} \mathrm{FeO}_{3}(x=0 ; 0.05 ; 0.1 ; 0.15 ; 0.2)$, полученной методом холодного прессования нанопорошка и последующим спеканием керамики при температуре $600^{\circ} \mathrm{C}$. $X$-ray дифракция показала формирование в составе с $x=0.1$ наряду с ромбоэдрической $R 3 c$ фазой орторомбической Pbam фазы. Увеличение концентрации Sm при замещении привело к структурным искажениям наряду с ростом диэлектрической проницаемости и уменьшением $\operatorname{tg} \delta$. Все образцы демонстрируют сильную дисперсию и большую диэлектрическую аномалию при $300^{\circ} \mathrm{C}$. Рост концентрации Sm приводит к структурно фазовому переходу из полярной $R 3 c$ фазы в антиполярную Pbam фазу с увеличением диэлектрической проницаемости.

\section{Финансирование работы}

Работа выполнена при финансовой поддержке государственного задания № 3.5982.2017/8.9.

\section{Конфоликт интересов}

Авторы заявляют, что у них нет конфликта интересов.

\section{Список литературы}

[1] W. Eerenstein, N.D. Mathur, J.F. Scott. Nature 442, 759 (2006).

[2] G. Catalan, J.F. Scott. Adv. Mater. 21, 2463 (2009).

[3] N. Hur, S. Park, P.A. Sharma. Nature 429, 392 (2004).

[4] Ch.-H. Yang, D. Kan, I. Takeuchi, V. Nagarajan, J. Seidel. Phys. Chem. Chem. Phys. 14, 15953 (2012)

[5] A. Hussain, X. Xu, G. Yuan, Y. Wang, Y. Yang, J. Yin, J. Liu, Z. Liu. Chin. Sci. Bull. 59, 36, 5161 (2014).

[6] H. Singh, K.L. Yadav. Ceram. Int. 41, 9285 (2015).

[7] K.P. Remya, S. Amirthapandian, M. Manivel Raja, C. Viswanathan, N. Ponpandian. J. Appl. Phys. 120, 134304 (2016). 
[8] Min Zhong, N. Pavan Kumar, E. Sagar, Zhu Jian, Hu Yemin, P. Venugopal Reddy. Chem. Phys. 173, 126 (2016).

[9] S. Pattanayak, R.N.P. Choudhary, P.R. Das. J. Adv. Dielect. 4, 1450011 (2014).

[10] Zhu Jian, N. Pavan Kumar, Min Zhong, Hu Yemin, P. Venugopal Reddy. J. Magn. Magn. Mater. 386, 92 (2015).

[11] Z.X. Cheng, A.H. Li, X.L. Wang, S.X. Dou, K. Ozawa, H. Kimura, S.J. Zhang, T.R. Shrout. J. Appl. Phys. 103, 07E507 (2008).

[12] B. Stojadinović, Z. Dohčević-Mitrović, N. Paunović, N. Ilić, N. Tasić, I. Petronijević, D. Popović, B. Stojanović. J. Alloys Comp. 657, 866 (2016).

[13] H. Dai, Z. Chen, T. Li, Y. Li. J. Rare Earths 30, 11, 1123 (2012).

[14] M.A. Ahmed, S.F. Mansour, S.I. El-Dek, M.M. Karamany. J. Rare Earths 34, 5, 495 (2016).

[15] D.V. Karpinsky, I.O. Troyanchuk, A.L. Zheludkevich, O.V. Ignatenko, M.V. Silibin, V.V. Sikolenko. Phys. Solid State 58, 8, 1590 (2016).

[16] B. Deka, S. Ravi, D. Pamu. Ceram. Int. 43, 18, 16580 (2017).

[17] P. Godara, A. Agarwal, N. Ahlawat, S. Sanghi. J. Molec. Structure 1097, 207 (2015).

[18] Ling Li, Hongwei Qin, Ling Zhang, Jifan Hu. RSC Adv. 6, 60967 (2016).

[19] S. Chaturvedi, P. Shyam, A. Apte, J. Kumar, A. Bhattacharyya, A.M. Awasthi, S. Kulkarni. Phys. Rev. B 93, 174117 (2016).

[20] S. Bhardwaj, J. Paul, S. Chand, K.K. Raina, R. Kumar, J. Mater, Sci.: Mater. Electron. (2014) 25:4568-4576.

[21] E. Palaimiene, J. Macutkevic, D.V. Karpinsky, A.L. Kholkin, J. Banys. Appl. Phys. Lett. 106, 012906 (2015).

[22] M. Polomska, W. Kaczmerk, Z. Pajak. Phys. Status Solidi A 23, 567 (1974).

[23] X. Marti, P. Ferrer, J. Herrero-Albillos, J. Narvaez, V. Holy, N. Barrett, M. Alexe, G. Catalan. Phys. Rev. Lett. 106, 236101 (2011).

[24] Cheng-Sao Chen, C.S. Tu, P.Y. Chen, V.H. Schmidt, Z.R. Xu, Yi Ting. J. Alloys. Comp. 687, 442 (2016).

[25] A. Kumar, J.F. Scott, R.S. Katiyar. Phys. Rev. B 85, 224410 (2012).

Редактор Т.Н. Василевская 